\title{
Interdisciplinaridade e Teoria de Redes: rede semântica de cliques baseada em ementas
}

\author{
Júlia Carvalho Andrade ${ }^{1}$, Renata Souza Freitas Dantas Barreto ${ }^{2}$, Núbia Moura \\ Ribeiro ${ }^{1}$, Hernane Borges de Barros Pereira ${ }^{1,2}$ \\ ${ }^{1}$ Doutorado Multi-Institucional e Multidisciplinar em Difusão do Conhecimento - \\ Universidade Federal da Bahia (UFBA) - Salvador - BA - Brasil \\ ${ }^{2}$ Programa de Pós-Graduação em Modelagem Computacional e Tecnologia Industrial \\ (SENAI-CIMATEC) - Salvador - BA - Brasil \\ juliacarvalhoandrade@yahoo.com.br, \{nubiamouraribeiro, \\ renatasouzabarreto, hernanebbpereira\}@gmail.com
}

\begin{abstract}
This work proposes to investigate the semantic networks of cliques based to the courses descriptions of a doctoral postgraduate program. A total of 46 courses descriptions were compiled. Using some indexes of social and complex networks as a starting point, it was observed that the centralities of degree, closeness and betweeness are adequate indexes to perceive the coherence and consistency of the proposal of a program with its set of courses descriptions. And the identification of the common topics in the courses descriptions helps in the perception of the interactions between the courses of a program.
\end{abstract}

Resumo. Este trabalho propõe investigar a rede semântica de cliques baseada em ementas de um programa de pós-graduação de doutorado. Foram coletadas 46 ementas de componentes curriculares. Usando alguns índices de redes sociais e complexas como ponto de partida, observou-se que as centralidades de grau, proximidade e intermediação são indices adequados para perceber a coerência e consistência da proposta de um programa com seu ementário. E a identificação dos temas em comum às ementas auxilia na percepção das interações entre os componentes curriculares de um programa.

\section{Introdução}

Os componentes curriculares da matriz curricular de um programa de pós-graduação (PPG) têm como objetivo proporcionar os conhecimentos necessários ao aprendizado proposto pelo PPG. Cada componente curricular possui uma ementa, que consiste em uma descrição discursiva e resumida do seu conteúdo teórico (conceitual) ou teóricometodológico (conceitual/procedimental). Neste sentido, o ementário de um PPG pode ser considerado um discurso escrito, o qual deve refletir sua proposta para o aprendizado. Ademais, cada ementa pode ser considerada uma sentença do discurso e o universo de palavras das ementas representa o vocabulário do discurso do PPG.

No Brasil, os programas de pós-graduação são avaliados e reconhecidos pela Coordenação de Aperfeiçoamento de Pessoal de Nível Superior (CAPES), e classificados em áreas de concentração, dentro de áreas de avaliação. Segundo a 
CAPES (2013), a estrutura curricular de um PPG deve ser adequada à formação de mestres e doutores, e constituída por um conjunto de componentes curriculares congruente com as áreas de concentração e as linhas de pesquisa. Desta forma, os textos descritivos das áreas de concentração e linhas de pesquisa de um PPG devem refletir sua proposta de aprendizado e espera-se coerência e consistência com seu ementário.

Para avaliar a coerência e a consistência entre a proposta de aprendizado baseada nas áreas de concentração e nas linhas de pesquisa de um PPG e o ementário relativo à sua matriz curricular, este estudo propõe a modelagem do ementário de um PPG em nível de doutorado, por meio de uma rede semântica de cliques baseada em ementas e o uso de temas-chave (i.e. conceitos representativos da proposta de aprendizado do PPG). Vale salientar que redes semânticas de cliques baseadas em ementas ainda não foram descritas na literatura científica até o presente momento.

\section{Trabalhos Correlatos}

Rede semântica é um sistema de representação do conhecimento de discursos. As redes semânticas de cliques é um tipo de rede semântica em que todas as palavras de cada sentença do discurso (e.g. texto) se conectam formando um subgrafo, chamado de clique. Assim, uma clique é um subgrafo completo de um grafo $G$. E as cliques se conectam formando a rede semântica do discurso [Fadigas e Pereira 2013].

As redes de cliques são formadas por justaposição e/ou sobreposição de cliques. O processo de justaposição significa conectar duas cliques com apenas um vértice em comum. E quando dois ou mais vértices conectam as cliques ocorre o processo de sobreposição [Fadigas e Pereira 2013], conforme ilustrado na Figura 1.

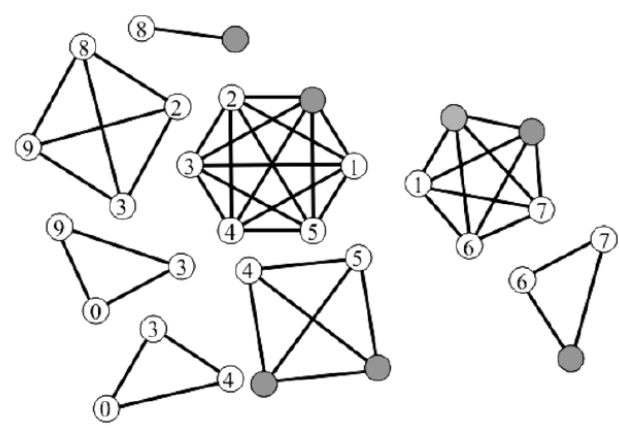

a) Estado inicial de cliques isoladas.

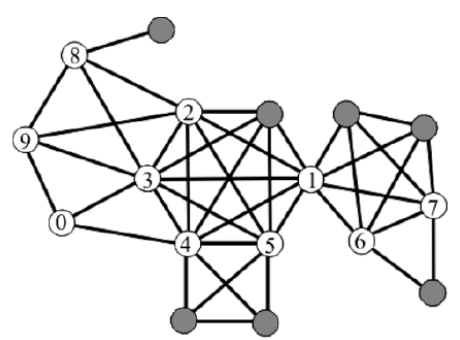

b) Uma "rede de cliques" conectada por justaposição e sobreposição.

Figura 1. Estado inicial de cliques isoladas e uma possível "rede de cliques". Fonte: Modificada de Fadigas e Pereira (2013, p. 2577).

Diversos trabalhos investigaram discursos por meio de redes semânticas de cliques. Caldeira et al. (2006) utilizaram redes de palavras baseadas em cliques para analisar a estrutura de conceitos significativos em textos (i.e. discursos) escritos. Redes semânticas baseadas em títulos de artigos de periódicos científicos (RST) foram estudadas por: Fadigas et al. (2009) que analisaram RST de divulgação em educação matemática; Pereira et al. (2011) estudaram a estrutura topológica de RST como um método de análise da eficiência da difusão da informação; e Henrique et al. (2014) utilizaram RST para comparar os títulos de artigos de periódicos de divulgação em 
educação matemática, em inglês e português. Já Teixeira et al. (2010), Lopes et al. (2015) e Lima-Neto et al. (2018) utilizaram redes semânticas de cliques para analisar a relação entre as palavras que emergem em discursos orais.

Ademais, Fadigas e Pereira (2013) propuseram um conjunto de índices para capturar as propriedades de redes semânticas de cliques e um método para caracterizar o fenômeno mundo pequeno nestas redes, utilizando como fonte de dados RST. E Grilo et al. (2017) investigaram a robustez de redes semânticas de cliques.

\section{Metodologia}

O fluxograma do processo metodológico é apresentado na Figura 2.

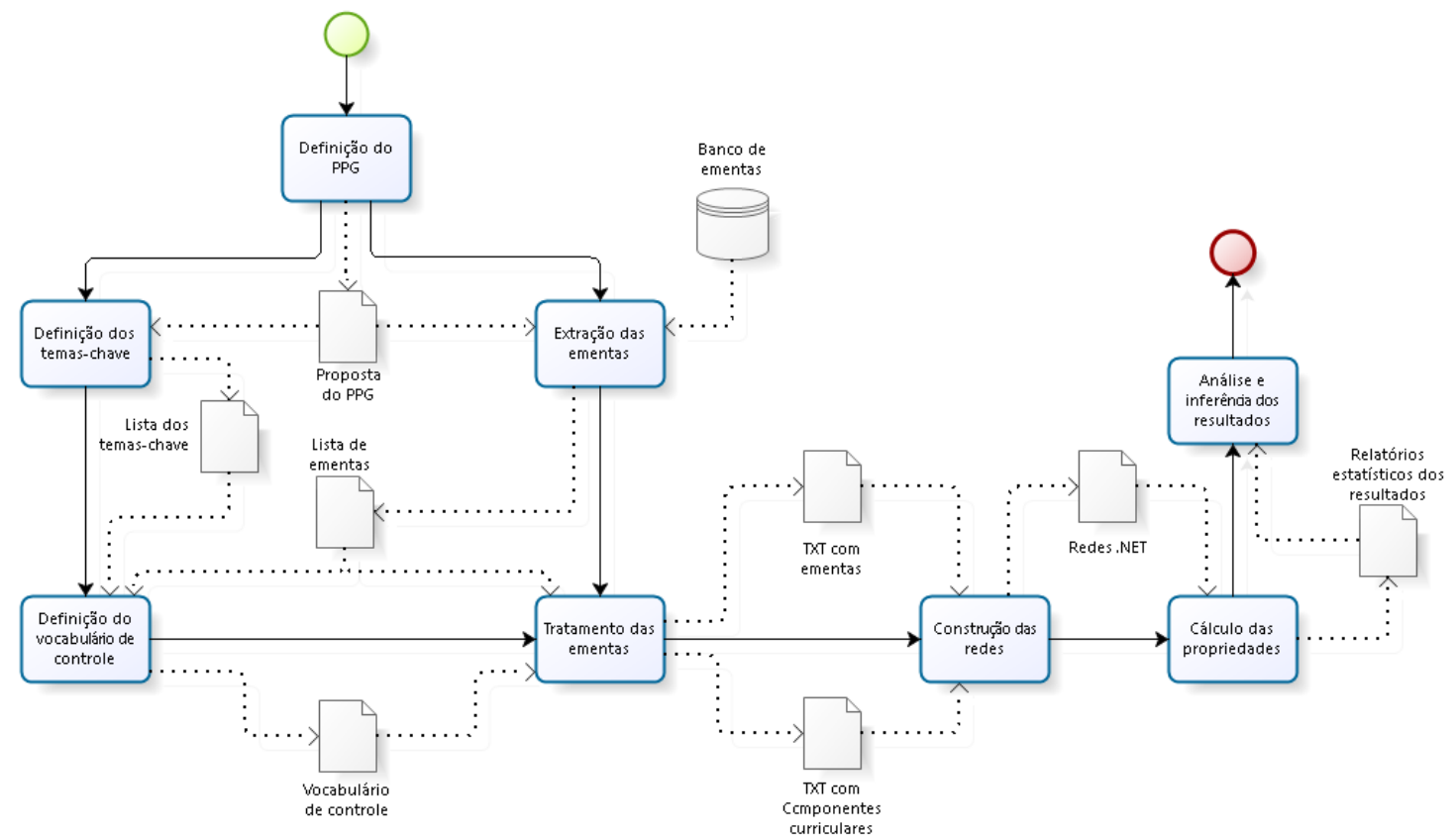

Figura 2: Fluxograma do processo metodológico. Fonte: Autores.

Para modelagem da rede de ementas foi definido como cenário um PPG, em nível de doutorado, da área de avaliação interdisciplinar da CAPES. O corpus de análise da sua proposta é constituído pelo texto descritivo da sua área de concentração "Modelagem da Geração e Difusão do Conhecimento" e pelos títulos das três linhas de pesquisa - 1. Construção do Conhecimento: Cognição, Linguagens e Informação / 2. Difusão do Conhecimento: Informação, Comunicação e Gestão / 3. Cultura e Conhecimento: Transversalidade, Interseccionalidade e (In)formação. Estas informações foram obtidas na Plataforma Sucupira. E o ementário com 46 ementas foi disponibilizado pelo colegiado do PPG estudado, que autorizou a pesquisa por meio da assinatura de Carta de Anuência. Vale salientar que este trabalho foi apreciado e aprovado pelo Comitê de Ética em Pesquisa da Escola de Nutrição da Universidade Federal da Bahia (Número do parecer: 2.487.366).

Os processos "Definição dos temas-chave" e "Definição do vocabulário de controle" (Figura 2) foram realizados de forma manual, seguindo as etapas: (1) Préanálise do corpus a partir da leitura para familiarização com o texto descritivo da área de concentração, títulos das linhas de pesquisa e ementas; (2) Identificação dos temas- 
chave no texto descritivo da área de concentração e nos títulos das linhas de pesquisa que representassem o conteúdo teórico ou teórico-metodológico da proposta de aprendizagem do PPG investigado; (3) Identificação de palavras relacionadas semanticamente aos temas-chave nas ementas; (4) Quantificação da frequência de aparição das palavras relacionadas aos temas-chave nas ementas a fim de selecionar aquelas de maior ocorrência, que foram escolhidas para representar os temas-chave.

Após essas etapas, foram identificados 15 temas-chave e palavras relacionadas que compõem o vocabulário de controle usado no tratamento das ementas (Tabela 1). Cabe ressaltar que os temas-chave "conhecimento", "construção do conhecimento", "difusão do conhecimento" e "geração do conhecimento", apesar de estarem estreitamente relacionados entre si, possuem significados distintos.

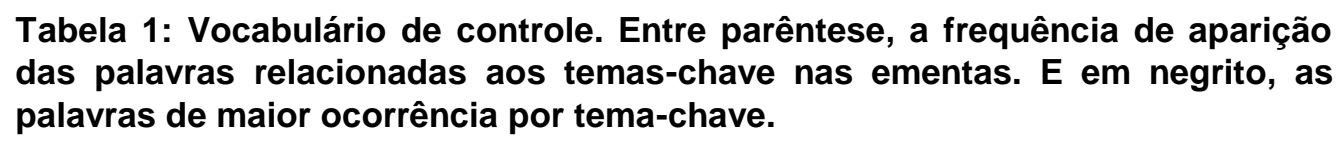

\begin{tabular}{|c|c|c|}
\hline \multicolumn{2}{|c|}{ TEMAS-CHAVE (T) } & PALAVRAS RELACIONADAS \\
\hline $\mathrm{T} 1$ & Metodologia & $\begin{array}{l}\text { Método (7), metodologias (2), aspectos metodológicos (1), } \\
\text { metodologias participativas (1), recorte teórico-metodológico (1) }\end{array}$ \\
\hline $\mathrm{T} 2$ & Cognição & $\begin{array}{l}\text { Análise cognitiva (2), ciências cognitivas (2), analista cognitivo (1), } \\
\text { cognição (1), cognólogo (1), processos cognitivos (1) }\end{array}$ \\
\hline T3 & Complexidade & Complexidade (5), redes complexas (1), sistemas complexos (1) \\
\hline $\mathrm{T} 4$ & Comunicação & $\begin{array}{l}\text { Comunicação (2), canais de comunicação (1), cenário comunicativo } \\
\text { (1), comunicação não verbal (1), relaçôes comunicativas (1) }\end{array}$ \\
\hline T5 & Ciência & $\begin{array}{l}\text { Ciência (7), desenvolvimento científico (1), difusão da ciência (1), } \\
\text { disseminação da ciência (1), divulgação da ciência (1), invenção (1), } \\
\text { científica (1), pesquisa científica (1), popularização da ciência (1), } \\
\text { produção científica (1) }\end{array}$ \\
\hline T6 & Tecnologia & $\begin{array}{l}\text { Tecnologia (4), desenvolvimento tecnológico (1), dimensão } \\
\text { tecnológica (1), inovação tecnológica (1), tecnologias da comunicação } \\
\text { (1), tecnologias da informação (2), transferência de tecnologia (1) }\end{array}$ \\
\hline $\mathrm{T} 7$ & Conhecimento & $\begin{array}{l}\text { Conhecimento (11), gestão do conhecimento (3), padronização do } \\
\text { conhecimento (2), bases de conhecimento (1), campos de conhecimento } \\
\text { (1), controle do conhecimento (1), disseminação do conhecimento (1), } \\
\text { divulgação do conhecimento (1), popularização do conhecimento (1) }\end{array}$ \\
\hline $\mathrm{T} 8$ & $\begin{array}{l}\text { Construção do } \\
\text { conhecimento }\end{array}$ & Construção do conhecimento (3) \\
\hline T9 & Cultura & $\begin{array}{l}\text { Cultura (4), cibercultura (2), abordagens culturais (1), antropologia } \\
\text { cultural (1), contextos culturais (1), cultura brasileira (1), culturalismo } \\
\text { (1), diversidade cultural (1), indústria cultural (1), matrizes culturais } \\
\text { (1), pluralismo cultural (1), politicas culturais (1), produção cultural (1) }\end{array}$ \\
\hline $\mathrm{T} 10$ & $\begin{array}{l}\text { Difusão do } \\
\text { conhecimento }\end{array}$ & Difusão do conhecimento (11) \\
\hline T11 & Epistemologia & $\begin{array}{l}\text { Epistemologia (1), bases epistemológicas (1), correntes } \\
\text { epistemológicas (1), escolhas epistemológicas (1) }\end{array}$ \\
\hline T12 & $\begin{array}{l}\text { Geração do } \\
\text { conhecimento }\end{array}$ & Geração do conhecimento (2) \\
\hline T13 & Informação & Informação (3), disseminação da informação (1) \\
\hline T14 & Linguagem & Linguagem (5) \\
\hline T15 & Modelagem & Modelo (9), modelagem (3), modelagem computacional (2) \\
\hline
\end{tabular}


$\mathrm{Na}$ construção de uma rede semântica de cliques baseada em ementas, cada ementa é considerada uma sentença de um discurso escrito, que, por sua vez, forma uma clique. As palavras de cada ementa são os vértices de uma clique e as arestas são as conexões entre as palavras que aparecem na mesma ementa. As palavras em comum às ementas são os vértices em comum às cliques, que fazem a justaposição e sobreposição das cliques, formando, assim, a rede de ementas (Figura 3).

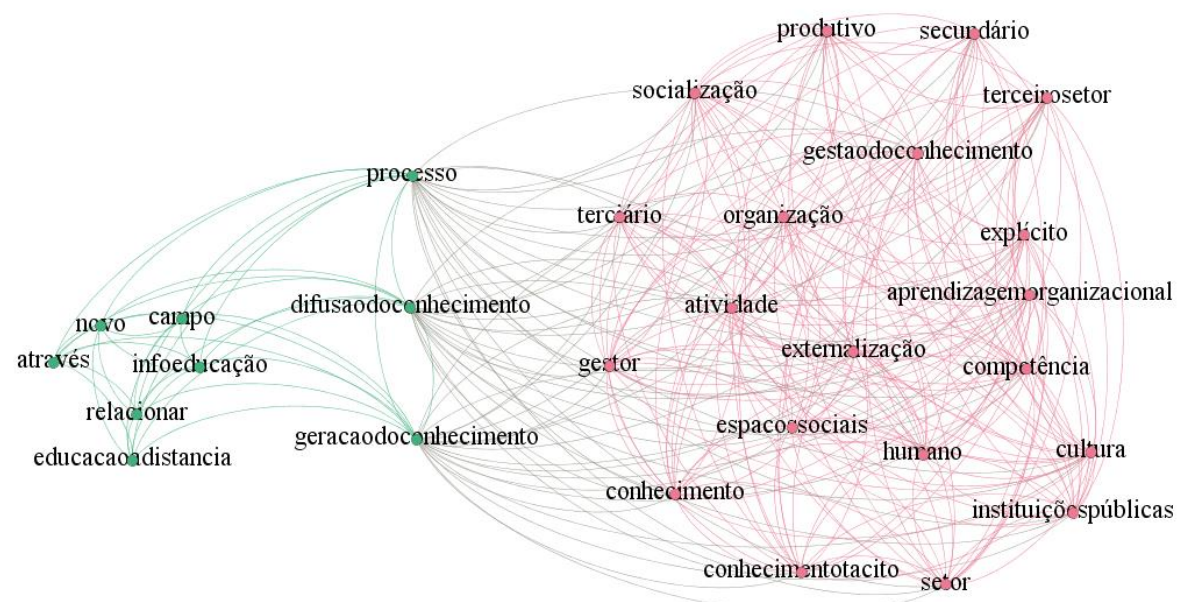

Figura 3: Excerto de uma rede de ementas. Fonte: Autores.

A Figura 3 mostra a rede semântica de cliques resultante das ementas das disciplinas "EDCA93 - Gestão do Conhecimento e Aprendizagem Colaborativa" e "EDCA94 - Infoeducação e Educação à Distância". As palavras "processo", "difusão do conhecimento" e "geração do conhecimento" são as palavras em comuns às ementas, que atuam como vértices de conexão fazendo a sobreposição entre as duas cliques.

Os processos de "Tratamento das ementas" e "Construção da rede semântica de cliques baseada em ementas" (Figura 2) foram realizados conforme as etapas a seguir:

(1) União das ementas em um único discurso, sendo cada ementa inserida em uma linha em um arquivo de formato .txt.

(2) Tratamento manual das ementas, que consistiu na aplicação de 11 regras gerais propostas por Pereira et al. (2011) e aplicação de uma nova regra devido a uma especificidade deste estudo, a saber: Em alguns casos, foi necessária a introdução de palavras de maior ocorrência por temas-chave nas ementas que tratavam dos temaschave, mas que não as continham. Isto propiciou a conexão entre as cliques que tratavam do mesmo tema-chave.

Por exemplo, havia ementas que explanavam sobre vários tipos de métodos (e.g. método analítico simplex, método de Householder e métodos estocásticos), e, seguindo o tratamento manual, estas palavras foram convertidas em "métodoanalíticosimplex", "métododehouseholder" e "métodosestocásticos", respectivamente. Como neste caso não aparecia a palavra "método" sozinha na ementa, a clique não iria se conectar a outras que tratassem do tema "T1: Metodologia" (Tabela 1), fazendo-se necessária a aplicação desta nova regra. 
Outra situação para aplicação dessa regra ocorre quando a ementa apresenta pelo menos uma palavra relacionada ao tema-chave, segundo o vocabulário de controle (Tabela 1), mas não apresenta a palavra de maior ocorrência, sendo esta introduzida na ementa com o intuito de proporcionar a conexão das cliques por temas-chave.

(3) Após essa etapa, foi feito o tratamento computacional das ementas. As ementas passaram por um conjunto de programas da UNITEX [Paumier 2008] para classificação, modificação e eliminação, quando necessária, das palavras. Também foi utilizado o conjunto de ferramentas computacionais desenvolvido por Caldeira et al. (2005), que permite, dentre outras coisas, identificar a classe gramatical de cada palavra utilizada na criação da rede. Ao final do tratamento computacional foram gerados dois arquivos de formato .txt: um contendo o vocabulário e a classificação gramatical das palavras; e outro com os pares de palavras, frequência de aparição das palavras e dos pares nas ementas, o qual permite identificar os vértices em comum às cliques.

(4) Um arquivo no formato .net, também gerado na etapa anterior, foi utilizado para construção e análise da rede de ementas no software Gephi, versão 0.9.1. Para caracterizar a rede, foram empregados os índices: número de vértices $(n=|V|)$, número de arestas $(m=|E|)$, grau médio $(<k>)$, diâmetro $(D)$, coeficiente de aglomeração médio $(C)$, caminho mínimo médio $(L)$ e densidade $(\Delta)$. Estes índices foram calculados para a rede de ementas e para a rede aleatória correspondente, a qual foi construída para caracterização topológica da rede de ementas. A rede aleatória foi gerada com o mesmo número de vértices e grau médio da rede de ementas. $\mathrm{E}$ os índices empregados para medir a importância dos vértices foram: centralidade de grau $(C G)$, centralidade de proximidade $(C P)$ e centralidade de intermediação $(C I)$.

\section{Resultados e Discussão}

Nesta rede de ementas, os vértices são as palavras empregadas nas ementas para descrever o conteúdo teórico (conceitual) ou teórico-metodológico (conceitual/ procedimental) dos componentes curriculares. Desta forma, os vértices representam conceitos e as arestas representam as conexões entre estes conceitos. Para reforçar essa afirmação, em revisão sistemática sobre redes semânticas, Rosa (2016) observou que, para a maioria dos estudos analisados $(80 \%)$, um vértice de uma rede semântica representa conceitos ou objetos e as arestas não dirigidas de uma rede semântica representam relações entre estes conceitos ou objetos. Considerar os vértices da rede semântica de ementas como conceitos é importante na interpretação dos índices de centralidade de proximidade e intermediação, a seguir.

A rede de ementas do PPG investigado é constituída por 766 vértices e 17.201 arestas. É considerada esparsa $(\Delta=0,059)$ e as palavras estão conectadas em média a aproximadamente 45 outras palavras $(\langle k\rangle=44,91)$. Isto sugere que as ementas possuem um vocabulário restrito para expressar os conhecimentos pretendidos.

A densidade é a razão entre o número de arestas existentes e o número máximo de arestas de uma dada rede. Em geral, percebe-se que a densidade da rede de ementas é maior que as observadas em RST (e.g. Pereira et al. 2016). Isto ocorre pelo fato de haver menor diversidade de palavras nas ementas do que entre as palavras dos títulos de artigos científicos. Quanto mais justaposições e sobreposições ocorram, maior densidade e maior coesão entre as ementas. 
O coeficiente de aglomeração é um índice que mede o quanto os vértices vizinhos de um vértice estão conectados entre si. No caso da rede investigada, o coeficiente de aglomeração médio é considerado alto $(C=0,92)$, o que indica que os vizinhos dos vértices têm muitos vizinhos entre si, havendo muitas conexões entre os vértices. Isto pode ser explicado pelo próprio método de construção de redes semânticas de cliques e também pela grande quantidade de vértices em comum às ementas. Assim, o coeficiente de aglomeração médio alto pode ser um indicativo de menor diversidade de palavras e maior ocorrência de justaposição e sobreposição.

O diâmetro da rede investigada é igual a quatro $(D=4)$. O caminho mínimo médio informa que, em média, a distância entre as palavras é de 2,302. I, o que significa que as palavras são muito próximas. Isto acontece pela quantidade de justaposições e sobreposições entre as ementas, refletindo no maior compartilhamento de conceitos em comum, o que pode ser um indício de interdisciplinaridade.

Quanto à caracterização topológica, pode-se afirmar que a rede de ementas $(R E)$ apresenta o fenômeno mundo pequeno, já que possui coeficiente de aglomeração médio elevado em relação ao da rede aleatória $(R A)$ correspondente $\left(C_{R E}=0,92>>C_{R A}\right.$ $=0,03)$ e caminho mínimo médio similar ao da rede aleatória correspondente $\left(L_{R E}=\right.$ 2,302 $\sim L_{R A}=2,382$ ), segundo o método de Watts e Strogatz (1998). Além disto, também foram observadas outras condições necessárias para que a rede investigada fosse considerada rede de mundo pequeno [Watts 1999], a saber: não dirigida, não ponderada, simples, esparsa e conectada (i.e. possui apenas um componente).

Considerando a análise de redes sociais, investigou-se também a importância dos vértices por meio dos índices de centralidade. As treze palavras da rede de ementas mais importantes em termo de centralidade de grau, em ordem decrescente, são: "conhecimento", "teoria", "abordagem", "difusão do conhecimento", "sociedade", "análise", "processo", "complexidade", "pesquisa", "aplicação", "rede", "método" e "modelo". Dentre estas, cinco representam temas-chave, e as outras, apesar de conceitualmente não representarem a proposta do PPG investigado, possuem significados relevantes para um curso de doutorado.

Tabela 2: Medidas de centralidades dos vértices que são as palavras de maior ocorrência por temas-chave.

\begin{tabular}{|l|c|c|c|}
\hline \multicolumn{1}{|c|}{ TEMAS-CHAVE: VÉRTICES } & $C G$ & $C P$ & $C I$ \\
\hline T7: Conhecimento & 277 & 0,6105 & 0,0720 \\
\hline T10: Difusão do conhecimento & 240 & 0,5930 & 0,0508 \\
\hline T3: Complexidade & 210 & 0,5795 & 0,0306 \\
\hline T1: Método & 190 & 0,5709 & 0,0585 \\
\hline T15: Modelo & 179 & 0,5429 & 0,0353 \\
\hline T5: Ciência & 150 & 0,5488 & 0,0172 \\
\hline T14: Linguagem & 143 & 0,5422 & 0,0305 \\
\hline T9: Cultura & 132 & 0,5387 & 0,0125 \\
\hline T11: Epistemologia & 89 & 0,5222 & 0,0000 \\
\hline T13: Informação & 76 & 0,4713 & 0,0054 \\
\hline T8: Construção do conhecimento & 67 & 0,4926 & 0,0021 \\
\hline T4: Comunicação & 60 & 0,4468 & 0,0022 \\
\hline T6: Tecnologia & 48 & 0,4885 & 0,0031 \\
\hline T12: Geração do conhecimento & 29 & 0,4279 & 0,0003 \\
\hline T2: Análise cognitiva & 25 & 0,4540 & 0,0003 \\
\hline
\end{tabular}


Do ponto de vista da importância dos vértices de maior ocorrência por temaschave nas ementas, pode-se observar que o vértice de maior centralidade de grau é "conhecimento" ( $C G=277)$, seguido pelo vértice "difusão do conhecimento" ( $C G=$ 240) (Tabela 2). Parece razoável afirmar que este fato é resultante da própria área de concentração em que o PPG está inserido, indicando assim que existe coerência e consistência entre a proposta do PPG e suas ementas em relação aos temas-chave "T7: Conhecimento" e "T10: Difusão do conhecimento".

Por outro lado, as palavras "análise cognitiva" $(C G=25)$ e "geração do conhecimento" ( $C G=29)$ são os vértices que tiveram menor centralidade de grau e ambas aparecem em apenas duas ementas (Tabela 2). Esta constatação sugere que esses são os temas-chave menos abordados pelos componentes curriculares em comparação aos outros. Porém, deveriam ganhar maior destaque nas ementas, uma vez que "análise cognitiva" é o tema-chave central da linha de pesquisa três e a "geração do conhecimento" é almejada pela área de concentração do PPG investigado.

A centralidade de proximidade está relacionada com a distância total de um vértice em relação a todos os demais vértices do grafo [Freeman 1979]. Na rede de ementas, os vértices de maior centralidade de proximidade são os conceitos mais próximos, mais centrais aos outros no conjunto de ementas. Na rede investigada, os conceitos mais próximos aos outros são "conhecimento" $(C P=0,6105)$ e "difusão do conhecimento" ( $C P=0,5930)$, respectivamente (Tabela 2$)$, o que já era de se esperar uma vez que estes vértices representam os temas-chave centrais do PPG investigado.

Já a centralidade de intermediação mede quantos caminhos mais curtos entre todos os pares de vértices do grafo passam por um determinado vértice [Freeman 1979]. Este índice avalia a frequência de ocorrência de um determinado vértice entre pares de outros vértices em caminhos mais curtos que os conectam. Na rede de ementas, os vértices com maior centralidade de intermediação são "conhecimento" $(C I=0,0720)$ e "método" $(C I=0,0585)$. Estes vértices estabelecem pontes entre vários outros conceitos na rede de ementas (Tabela 2).

$\mathrm{Na}$ rede de ementas foram observadas 10 comunidades com o índice de modularidade igual a 0,619 (Figura 4). Newman e Girvan (2004) definem comunidades como grupos de vértices densamente conectados. Em redes semânticas, os vértices que compõem as comunidades formam grupos de palavras bem integrados ou semanticamente relacionados. Este fato também é observado em redes semânticas de cliques, mas, não necessariamente, as comunidades refletem as cliques, pois as justaposições e sobreposições entre as cliques aumentam a densidade das comunidades.

Observa-se na Figura 4 que a comunidade azul claro agrupa os seguintes vértices: "conhecimento", "difusão do conhecimento", "geração do conhecimento", "construção do conhecimento" e "cognição". Estas palavras possuem significados distintos, mas correlacionados entre si, e fazem parte do processo de "Modelagem da Geração e Difusão do Conhecimento", área de concentração do PPG investigado. Por isso, esta comunidade representa um importante núcleo semântico do PPG investigado. Também pode-se observar que os vértices que representam os temas-chave "difusão do conhecimento", "ciência", "complexidade" e "tecnologia" são vértices que atuam como "pontes" entre comunidades, ligando, assim, o que pode ser mais um indício da importância destes conceitos na promoção de interdisciplinaridade. 


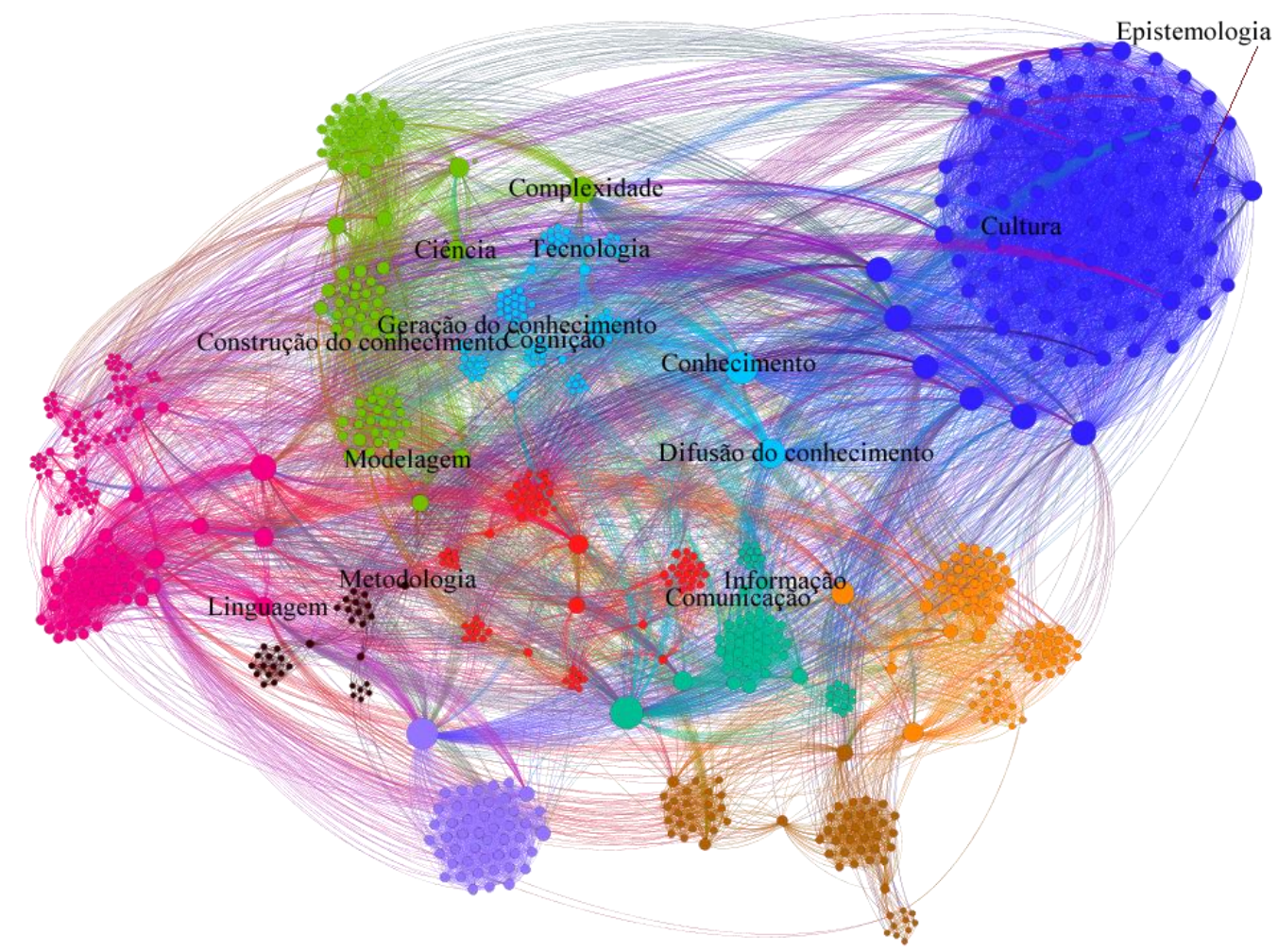

Figura 4. Rede de ementas, com destaque para os vértices de maior centralidade de grau e distribuição espacial por comunidades. Fonte: Autores.

A identificação dos temas-chave nas ementas permitiu agrupar os componentes curriculares por temas-chave (Tabela 3), e, a partir disto, foi construída a rede de componentes curriculares (Figura 5). Apesar da discussão sobre este tipo de rede estar fora do escopo deste artigo, far-se-á uma análise preliminar a seu respeito.

Tabela 3: Agrupamento dos componentes curriculares por temas-chave.

\begin{tabular}{|c|c|l|}
\hline \multicolumn{2}{|c|}{ TEMAS-CHAVE (T) } & COMPONENTES CURRICULARES \\
\hline T1 & Metodologia & EDCA91; EDCA86; EDCA98; EDCA99; EDCB04; EDCB09; EDCB76 \\
\hline T2 & Cognição & EDCE31; EDCC42 \\
\hline T3 & Complexidade & EDCA85; EDCA87; EDCA89; EDCB05; EDCB09 \\
\hline T4 & Comunicação & EDCB07; EDCB10 \\
\hline T5 & Ciência & EDCA85; EDCA89; EDCA92; EDCB05; EDCB06; EDCJ78; EDCE47 \\
\hline T6 & Tecnologia & EDCA90; EDCA92; EDCB06; EDCH10 \\
\hline T7 & Conhecimento & $\begin{array}{l}\text { EDCA85; EDCA88; EDCA89; EDCE30; EDCA92; EDCA93; } \\
\text { EDCB09; EDCC42; EDCB07; EDCJ78; EDCC42 }\end{array}$ \\
\hline T8 & $\begin{array}{c}\text { Construção do } \\
\text { conhecimento }\end{array}$ & EDCA89; EDCH68; EDCA92 \\
\hline T9 & Cultura & EDCE32; EDCB07; EDCB09; EDCA93 \\
\hline T10 & $\begin{array}{c}\text { Difusão do } \\
\text { conhecimento }\end{array}$ & $\begin{array}{l}\text { EDCA89; EDCA90; EDCA93; EDCA94; EDCA95; EDCB09; } \\
\text { EDCC42; EDCC51; EDCE32; EDCH10; EDCJ78 }\end{array}$ \\
\hline T11 & Epistemologia & EDCB09 \\
\hline T12 & $\begin{array}{c}\text { Geração do } \\
\text { conhecimento }\end{array}$ & EDCA93; EDCA94 \\
\hline T13 & Informação & EDCA90; EDCB07; EDCB10 \\
\hline T14 & Linguagem & EDCA96; EDCA97; EDCB10; EDCC49; FISB39 \\
\hline T15 & Modelagem & $\begin{array}{l}\text { EDCA85; EDCA88; EDCA89; EDCA91; EDCA92; EDCA95; } \\
\text { EDCB03; EDCB04; EDCB76 }\end{array}$ \\
\hline
\end{tabular}


$\mathrm{Na}$ Tabela 3, observa-se o agrupamento dos componentes curriculares por temas-chave (i.e. principais conceitos extraídos a proposta de aprendizagem do PPG investigado). Isto pode auxiliar os discentes na identificação de componentes curriculares importantes para o desenvolvimento de suas teses.

$\mathrm{Na}$ rede de componentes curriculares apresentada na Figura 5, o tamanho dos vértices é proporcional ao seu grau. Este índice indica a quantidade de componentes curriculares adjacentes com que cada um deles se relaciona por meio de conceitos em comum. O grau também sofre influência do peso das arestas. Neste estudo, foram atribuídos pesos às arestas, equivalentes à quantidade de temas-chave em comum às disciplinas. Os pares de vértices com maior peso são aqueles que possuem mais temaschave em comum. Por exemplo, os vértices "EDCA85 - Epistemologia e Construção do Conhecimento" e "EDCA89 - Processos de Construção do conhecimento" se conectam com peso quatro, uma vez que compartilham quatro temas-chave: "modelagem", "conhecimento", "complexidade" e "ciência" (Tabela 3).

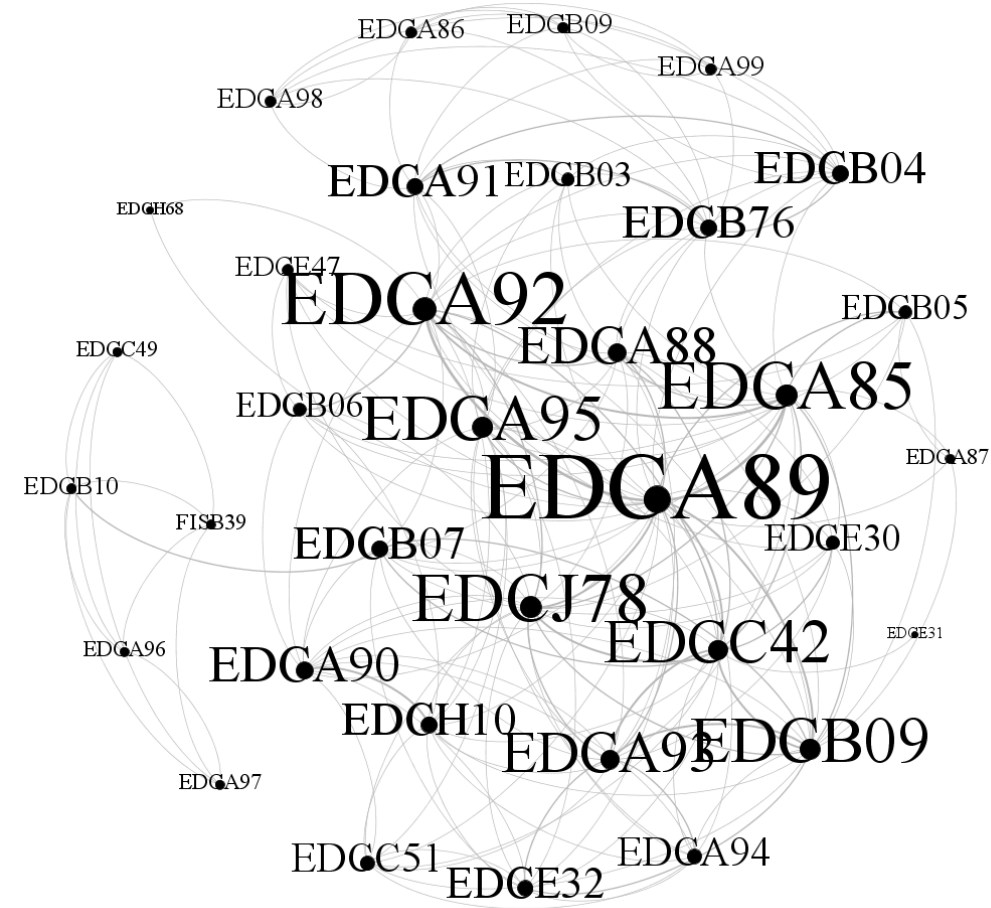

Figura 5. Rede de componentes curriculares do PPG investigado, com destaque para os vértices de maior centralidade de grau. Fonte: Autores.

Os vértices com as maiores centralidades de grau (EDCA89, EDCA85 e EDCA92) (Figura 5) são os componentes curriculares potenciais para a implementação de projetos de ensino interdisciplinaridade. Esta afirmação é corroborada pelo pressuposto que a interdisciplinaridade como uma abordagem de ensino propõe a interação entre duas ou mais disciplinas por meio de conceitos (e.g. temas-chave) compartilhados ou relacionados entre si. Segundo Japiassu e Marcondes (1993), a interdisciplinaridade consiste em um método de pesquisa e de ensino que possibilita que duas ou mais disciplinas interajam entre si, seja por meio da comunicação de ideias, integração mútua de conceitos, epistemologias, terminologias, metodologias, procedimentos e dados, ou pela forma de organização da pesquisa. 


\section{Considerações Finais}

A rede semântica de cliques baseada em ementas permitiu avaliar a coerência e a consistência entre a proposta de aprendizado baseada nas áreas de concentração e nas linhas de pesquisa de um PPG e o ementário relativo à sua matriz curricular.

Neste estudo, a coerência e a consistência confirmam-se apenas em relação a alguns dos 15 temas-chave. Os vértices "conhecimento" e "difusão do conhecimento" apresentaram os maiores valores de centralidade de grau, proximidade e intermediação, denotando sua importância nas ementas. Esta relevância, porém, não foi observada em relação aos vértices "análise cognitiva" e "geração do conhecimento". Além disso, foi constatado que os processos de justaposição e sobreposição impactam sobremaneira nos índices da rede semântica de cliques baseada em ementas repercutindo em suas características topológicas e na preeminência dos vértices.

A partir da identificação dos temas-chave nas ementas, foi possível agrupar os componentes curriculares por temas-chave e, a partir disto, construiu-se a rede de componentes curriculares. Este tipo de rede demostra a integração entre os componentes curriculares por meio de conceitos relevantes (i.e. temas-chave).

As redes de ementas e de componentes curriculares sugerem que a teoria de redes pode ser empregada para análise de conexões entre palavras das ementas e entre componentes curriculares da matriz curricular. A análise das conexões entre os conceitos pode auxiliar na busca de conceitos relacionados ao objeto de pesquisa dos discentes de forma mais eficiente e na identificação do vocabulário relacionado a estes conceitos. Já a análise das conexões entre os componentes curriculares pode auxiliar no estabelecimento de critérios de pré-requisitos entre eles.

Desta forma, sendo a teoria de redes uma ferramenta que auxilia no estudo de sistemas compostos por elementos que se conectam, auxiliando na visão da complexidade do todo, pode auxilia na percepção da complexidade de um PPG. Ademais, espera-se que este trabalho contribua para elaboração de um modelo computacional baseado na teoria de redes capaz de apoiar o planejamento e avaliação de programas de pós-graduação.

\section{Referências}

Caldeira, S. M. G. (2005) "Caracterização da rede de signos linguísticos: Um modelo baseado no aparelho psíquico de Freud". Mestrado Interdisciplinar em Modelagem Computacional, Fundação Visconde de Cairu, Salvador.

Caldeira, S. M. G., Petit Lobão, T. C., Andrade, R. F. S, Neme, A. e Miranda, J. G. V. (2006). The network of concepts in written texts. In The European Physical Journal $B$ - Condensed Matter and Complex Systems, 49(4):523-529.

Coordenação de Aperfeiçoamento de Pessoal de Nível Superior (CAPES). (2013) Diretoria de Avaliação. “Avaliação Trienal 2013”. In: Documento de Área. Área de Avaliação: Interdisciplinar.

Fadigas, I. S. e Pereira, H. B. B. (2013). A network approach based on cliques. In Physica A: Statistical Mechanics and its Applications, 392(10):2576 - 2587. 
Fadigas, I. S., Henrique, T., Pereira, H. B. B., Senna, V. e Moret, M. (2009). Análise de redes semânticas baseada em títulos de artigos de periódicos científicos: o caso dos periódicos de divulgação em educação matemática. In Educação Matemática Pesquisa, 11(1):167-193.

Freeman, L. C. (1979). Centrality in Social Networks: Conceptual clarification. In Social Networks, 1(3):215-239.

Grilo, M., Fadigas, I. S., Miranda, J. G. V., Cunha, M. V., Monteiro, R. L. S. e Pereira, H. B. B. (2017). Robustness in semantic networks based on cliques. In Physica. A (Print), 472:94-102.

Henrique, T., Fadigas, I. S., Rosa, M. G. e Pereira, H. B. B. (2014). Mathematics education semantic networks. In Social Network Analysis and Mining, 4:200.

Japiassu, H. e Marcondes, D. (1993) “Dicionário básico de filosofia”. In: Zahar, Rio de Janeiro.

Lima-Neto, J. L. A., Cunha, M. V. e Pereira, H. B. B. (2018). Redes semânticas de discursos orais de membros de grupos de ajuda mútua. In Obra Digital: Journal of Communication And Technology, 14: 51-66.

Lopes, C. R. S., Cardoso, J. P., Meira, S. S., Casotti, C. A., Vilela, A. B. A. e Pereira, H. B. B. (2015). Significado de corresidência na visão de idosos: uma estratégia para análise cognitiva com uso de redes semânticas. In Revista Saúde.com, 11:174-182.

Newman, M. E. J. e Girvan, M. (2004). Finding and evaluating community structure in networks. In Phys. Rev. E., 69(2):026113.

Paumier, S. (2008) UNITEX 2.0. “User Manual”, Université Paris-Est Marne-la-Vallée, http://unitexgramlab.org/releases/2.0/man/Unitex-GramLab-2.0-usermanual-en.pdf

Pereira, H. B. B, Fadigas, I., Senna, V. e Moret, M. (2011). Semantic networks based on titles of scientific papers. In Physica A: Statistical Mechanics and its Applications, 390(6):1192-1197.

Pereira, H. B. B, Fadigas, I. S., Monteiro, R. L. S., Cordeiro, A. J. A. e Moret, M. A. (2016). Density: A measure of the diversity of concepts addressed in semantic networks. In Physica. A (Print), 441:81-84.

Rosa, M. G. (2016) "Modelo empírico para analisar a robustez de redes semânticas". Doutorado Multidisciplinar e Multi-institucional em Difusão do Conhecimento. Universidade Federal da Bahia, Salvador.

Teixeira, G., Aguiar, M., Pereira, H., Miranda, J., Cunha, M., Morais, J., Carvalho, C. e Dantas, D. (2010). Complex semantics networks. In International Journal of Modern Physics C, 21(3):333-347.

Watts, D. J. (1999) "Small Worlds: The dynamics of Networks between Order and Randomness", Princetown University Press, New Jersey.

Watts, D. J. e Strogatz, S. H. (1998). Collective dynamics of small-world networks. In Nature, 393(4):440-442. 